\title{
Aproximación a la investigación en Didáctica de la Matemática
}

\author{
Luis Rico \\ Universidad de Granada (España)
}

Recibido el 16 de enero de 2012; aceptado el 22 de febrero de 2012

\section{Aproximación a la investigación en Didáctica de la Matemática}

\section{Resumen}

Objetivo de este documento es servir de introducción al joven investigador que se propone iniciar su especialización en Didáctica de la Matemática. Esta aproximación se hace desde la filosofía de la ciencia, la sociología de la ciencia y la metodología de la investigación. Usualmente, los expertos consideran varios componentes para una disciplina: el lenguaje, la estructura, el conocimiento, el significado y el método. Este trabajo presenta una reflexión sobre la Didáctica de la Matemática a partir de estos componentes. Educación matemática, comunidad de educadores matemáticos, ámbitos de trabajo, oficios de la investigación y otros términos, describen la conceptualización social de la disciplina y expresan su campo y ámbitos de acción. Para argumentar el carácter científico de Didáctica de la Matemática y precisar el estatus de su investigación desarrollamos algunos principios que organizan y muestran su estructura. Discutimos el significado de algunas nociones y concluimos con una revisión de aportes teóricos y metodológicos para la disciplina.

Palabras clave. Didáctica de la Matemática, investigación, disciplina científica, educación matemática, formación inicial de investigadores.

\section{Abordagen a pesquisa em Didática da Matemática}

\section{Resumo}

Objetivo deste documento destina-se como uma introdução para o jovem pesquisador que pretende lançar a sua especialização em Didática da Matemática. Esta abordagem é baseada na filosofia da ciência, da sociologia da ciência e da metodologia de pesquisa. Normalmente, os especialistas consideram vários componentes estruturais para uma disciplina: a linguagem, estrutura, conhecimento e método. Este artigo apresenta uma reflexão sobre o Didática da Matemática a partir desses componentes. Educação matemática, comunidade educativa de educadores matemáticos, áreas de trabalho, escritórios e outras pesquisas, são os termos que descrevem a disciplina e expressar seus campos e campos de ação. Para argumentar a natureza científica da Didática da Matemática e clarificar o estatuto de sua pesquisa, desenvolvemos alguns princípios que organizam e mostrar sua estrutura. Discutimos o significado de algumas noções e concluir com uma revisão das contribuições teóricas e metodológicas da disciplina.

Termos-chave. Didática da Matemática, disciplina a investigação científica, educação matemática, formação inicial de investigadores.

Para citar: Rico, L. (2012). Aproximación a la Investigación en Didáctica de la Matemática. Avances de Investigación en Educación Matemática, 1, 39 - 63 


\section{Aproach to the research on Didactics of Mathematics}

\section{Abstract}

The purpose of this paper is to provide an introduction to the young researcher who intends to initiate he/her specialization in Didactics of Mathematics. This approach is based on the philosophy of science, the sociology of science and the research methodology. Among the structural components that experts usually consider are: the language, structure, knowledge, meaning and method. This paper presents a reflection on the Didactics of Mathematics based on these components. Mathematics education, community of mathematics educators, work areas, researching, and others are the terms that describe the discipline and express their fields of action. To argue the scientific nature of Didactics of Mathematics and clarify the status of its research, we develop some principles to organize and show its structure. We discuss the meaning of some notions and we conclude with a review of theoretical and methodological contributions for the discipline.

Key words. Didactics of Mathematics, research, scientific discipline, mathematics education, initial training of researchers.

\section{Approche de la recherche en Didactique des Mathématiques}

\section{Résumé}

Objectif de ce document est conçu comme une introduction au jeune chercheur qui a l'intention de lancer sa spécialisation en Didactique des Mathématiques. Cette approche est basée sur la philosophie de la science, de la sociologie de la science et de la méthodologie de la recherche. Habituellement les experts considèrent plusieurs éléments structuraux pour une discipline: la langue, la structure, la connaissance et la méthode. Cet article présente une réflexion sur la Didactique des Mathématiques à partir de ces composants. Milieu de l'éducation de mathématiques, de professeurs de mathématiques, des aires de travail, les bureaux et autres travaux de recherche, sont les termes qui décrivent la discipline et d'exprimer leurs champs et des champs d'action. Pour argumenter le caractère scientifique de la Didactique des Mathématiques et de clarifier le statut de leurs recherches, nous avons développé certains principes qui organisent et montrent leur structure. Nous discutons de la signification de certaines notions et de conclure par un examen des apports théoriques et méthodologiques de la discipline.

Paroles clés. Didactique des Mathématiques, recherche, discipline scientifique, l'enseignement des mathématiques, formation initiale des chercheurs.

\section{Introducción}

Esta reflexión está dirigida a los jóvenes que se proponen iniciar su especialización en Didáctica de la Matemática, disciplina en la cual quieren formarse y en la que esperan alcanzar cierta competencia como investigadores.

\subsection{Perfil de los estudiantes}

El nivel de formación que tienen los estudiantes españoles aspirantes a investigar en Didáctica de la Matemática es, mayoritariamente, el grado en matemáticas ${ }^{1}$. A su vez, el nivel inicial que tienen los estudiantes que proceden de países latinoamericanos es el grado de matemáticas o de profesor de matemáticas, que varía según los distintos

\footnotetext{
${ }^{1}$ El grado es el nivel básico de especialización dentro de las titulaciones que organizan los estudios universitarios en España. Las enseñanzas de grado tienen como finalidad la especialidad en una o varias disciplinas para el ejercicio de actividades de carácter profesional.
} 
programas, universidades y países de procedencia. La preparación en Didáctica de la Matemática con que los estudiantes de grado acceden a un máster en esta especialidad es de iniciación, que puede alcanzar conocimientos generales, sin profundidad sobre temas específicos de la disciplina.

Los graduados que comienzan estudios de posgrado en Didáctica de la Matemática comparten una atracción y gusto personal hacia las matemáticas. También han percibido actitudes negativas hacia las matemáticas escolares, actitudes que rechazan y que se proponen superar.

Estos graduados están convencidos de que es posible salvar este conflicto y mejorar la práctica de la enseñanza y el aprendizaje de las matemáticas. Argumentan que los problemas que se detectan en el sistema educativo sobre las matemáticas son superables, una vez se conozcan sus causas. Sostienen que la planificación contribuye a optimizar el tiempo y los recursos, que la observación y la orientación de los escolares ayudan a promover su aprendizaje, que la evaluación fomenta la crítica y mejora el propio conocimiento. Consideran que la renovación de la enseñanza y el aprendizaje de las matemáticas en el aula es una empresa factible y necesaria, por ello confían en mejorarla desde un conocimiento especializado.

Estas cuestiones preocupan a los graduados, que se proponen ahondar en su comprensión. Resolver estos retos profesionales es lo que esperan de la investigación en Didáctica de la Matemática. Aunque la mayoría de ellos no tengan experiencia previa, mantienen la convicción de que la indagación en Didáctica de la Matemática dará solución a los problemas mencionados.

Si bien estas motivaciones están bien delimitadas, es frecuente que desconozcan la disciplina que aborda esos interrogantes y problemas de manera especializada. $\mathrm{Su}$ desconocimiento se extiende a la naturaleza de la Didáctica de la Matemática y a la discusión sobre su carácter científico, teórico, técnico o práctico. También desconocen la organización y funcionamiento de la comunidad de investigadores. Por ello resulta muy conveniente una aproximación a la disciplina que contribuya a su iniciación como investigadores en este nuevo campo.

\subsection{Campo de estudio}

Para abordar de manera profesional el campo de la Didáctica de la Matemática, el investigador novel requiere delimitar su ámbito de trabajo, necesita concretar la naturaleza de las cuestiones específicas que aborda, precisa reconocer y determinar las herramientas teóricas y metodológicas mediante las que plantear y definir tales interrogantes. Estas condiciones son apropiadas para que el estudiante de posgrado realice su especialización y desarrolle aquellas competencias profesionales que le cualificarán como investigador.

Objetivo principal de este documento es proporcionar al investigador novel un marco que le aproxime a la Didáctica de la Matemática, que conecte su incipiente formación previa con unas nociones y unos conceptos básicos sobre investigación en el área. 


\section{Inicio de la formación del investigador}

En el inicio de su formación como investigador conviene que el aspirante organice sus ideas para entender que la Didáctica de la Matemática forma parte del conocimiento científico y que, como disciplina, viene orientada por la investigación.

\subsection{Términos usuales}

Revisar los usos y significados de términos utilizados en este campo se puede realizar consultando diccionarios, bases de datos, libros de filosofía, de sociología o de historia de la ciencia. Así, por ciencia se entiende:

- "Conocimiento cierto de las cosas por sus principios y causas." (Real Academia Española [RAE], 1992)

- "Descripción y correlación de aspectos de lo real obtenidos mediante la observación, la abstracción y la lógica.” (Real Academia de Ciencias Exactas, Físicas y Naturales [RAC], 1990)

- "Conjunto organizado de conocimientos que versa sobre un objeto determinado, tiene un método propio y se funda en relaciones objetivas comprobables." (Seco, Andrés, \& Ramos, 1999, p. 1038)

Desde una perspectiva social de la ciencia interesa subrayar que, en la sociedad actual, la ciencia destaca como forma de conocimiento objetivo, fundado, verdadero y valioso. La investigación, como modo adecuado de proceder para la obtención de dicho conocimiento, se muestra como tarea respetable. Por su dimensión social la ciencia también se contempla como actividad dotada de poder transformador del mundo. Los condicionantes de la ciencia y la investigación como actividades sociales se han universalizado y extendido a todas las disciplinas, de ahí que gran parte de las prácticas profesionales hayan alcanzado la consideración de científicas (Echevarría, 1995).

Al inicio de su preparación resulta conveniente que el aspirante a investigador elabore un listado de nociones básicas junto con sus significados usuales, con las que construir una red de conceptos. Provisto de los significados de estas nociones generales y pensando con ellas, el investigador novel podrá abordar trabajos más especializados.

\subsection{Componentes de la Ciencia}

En el estudio de la filosofía de la ciencia los expertos consideran diversos componentes estructurales. Entre los componentes que, usualmente, se contemplan se encuentran: el lenguaje, la estructura, el conocimiento, el significado, el método, la actividad, los fines y los valores (González, 2010). Aportamos una reflexión a la Didáctica de la Matemática a partir de los primeros componentes, dejando pendientes los tres últimos para un trabajo próximo.

\section{El lenguaje}

Esta aproximación a la Didáctica de la Matemática se inicia con el lenguaje básico desde el cual se organizan sus significados generales. Estos términos son: educación matemática, comunidad de educadores matemáticos, ámbitos de trabajo, oficios de la investigación y otros, con los que describimos la disciplina y expresamos las cuestiones que aborda. 


\subsection{Educación matemática}

Para distinguir niveles de actividad y delimitar problemas elegimos dos términos centrales: educación matemática y Didáctica de la Matemática.

Concebimos la educación como "ese proceso mediante el cual un individuo en formación es iniciado en la herencia cultural que le corresponde" (Mead, 1985, p. 191), el modo en que cada generación transmite a las siguientes sus pautas culturales básicas; hace referencia a un sistema de valores, considera la práctica social en la que se incardina, se basa en unos fundamentos éticos y reflexiona sobre las implicaciones políticas conexas.

¿Qué entendemos por educación matemática?

Niños, adolescentes y jóvenes reciben parte importante de su herencia cultural a través de un sistema social de formación organizado, que se denomina sistema educativo. Las matemáticas forman parte de la cultura que transmite el sistema educativo y son parte esencial de la formación básica que han de compartir todos sus miembros; por ello tiene pleno sentido hablar de educación matemática. (...) La educación matemática abarca desde las primeras nociones sobre el número, la forma, el razonamiento, la prueba y la estructura que enseñamos a nuestros niños, hasta su culminación en una formación profesional o en estudios superiores. (...) Desde la perspectiva del especialista consideramos la educación matemática como conjunto de ideas, conocimientos y procesos implicados en la construcción, representación, transmisión y valoración del conocimiento matemático que tiene lugar con carácter intencional. La educación matemática que se transmite por medio del sistema escolar tiene rasgos epistémicos de actividad científica básica (...) También la actividad de los profesores y los procesos para su formación como profesionales quedan comprendidos dentro de la educación matemática (Rico, Sierra, \& Castro, 2000, pp. 352- 353).

\section{2. Ámbitos de trabajo en educación matemática}

Con educación matemática expresamos que nuestro foco de estudio se encuentra en las ciencias del hombre, establecemos su raíz antropológica y subrayamos su dimensión social.

En términos generales, establecemos tres sentidos distintos para educación matemática. En primer lugar, educación matemática como conjunto de conocimientos, artes, destrezas, lenguajes, convenciones, actitudes y valores, centrados en las matemáticas y que se transmiten por medio del sistema escolar. La educación matemática en este caso se refiere al conocimiento matemático como objeto de enseñanza y aprendizaje; la finalidad de la educación matemática se centra aquí en enriquecer y estructurar de manera adecuada los diversos significados de los conceptos matemáticos.

En segundo lugar, educación matemática como actividad social que tiene lugar en unas instituciones determinadas y se lleva a cabo por unos profesionales cualificados. En este caso se entiende la educación matemática como la totalidad de acciones y condiciones que hacen posible la enseñanza de las matemáticas, incluida la cualificación profesional de profesores. Abarca, pues, el conjunto de conocimientos, procesos y condiciones que posibilitan las interacciones entre profesores y alumnos y que hacen viable la enseñanza y aprendizaje de las matemáticas. En este caso se trata del saber y de las capacidades profesionales necesarias para transmitir y valorar el conocimiento matemático. 
En tercer lugar, como disciplina científica; es en este caso cuando nos referimos a la Didáctica de la Matemática.

La Didáctica de la Matemática se ocupa de indagar metódica y sistemáticamente los procesos de enseñanza y aprendizaje de las matemáticas así como los planes para la cualificación profesional de los educadores matemáticos. La Didáctica de la Matemática tiene como objeto delimitar y estudiar los fenómenos que se presentan durante los procesos de organización, comunicación, transmisión, construcción y valoración del conocimiento matemático (Rico, Sierra, \& Castro, 2000, pp. 353-354).

\subsection{Didáctica de la Matemática e investigación}

Como disciplina, la Didáctica de la Matemática tiene su campo de estudio en ese complejo de actividades de significación y tareas sociales de formación que hemos denominado educación matemática. Por ello decimos que la Didáctica de la Matemática se ocupa de indagar metódica y sistemáticamente sobre la enseñanza y aprendizaje de las matemáticas, sostener los planes para la cualificación profesional de los educadores matemáticos, y proporcionar fundamentación teórica y empírica.

La Didáctica de la Matemática, alias ciencia de la educación matemática, es el campo académico y científico de investigación y desarrollo que se propone identificar, caracterizar y entender los fenómenos y procesos, en potencia o en acto, implicados en la enseñanza y aprendizaje de las matemáticas de cualquier nivel educativo (Niss, 1998, pp. 4-5).

Esta es nuestra aproximación: surgen multitud de problemas teóricos y factuales en los procesos de comunicación, enseñanza y aprendizaje de las matemáticas, algunos de ellos de especial intensidad; también surgen problemas específicos en los procesos de formación y actualización de los educadores matemáticos. La Didáctica de la Matemática es aquella disciplina que se ocupa de estudiar e investigar los fenómenos y problemas de la educación matemática y proponer marcos explicativos mediante los cuales abordar su estudio y resolución (Rico, Sierra, \& Castro, 2000, pp. 356).

En Didáctica de la Matemática un trabajo de investigación se caracteriza mediante: su encuadre teórico, su marco metodológico y su ámbito de actuación.

\subsection{Comunidad de educadores matemáticos}

La consideración de la ciencia como actividad humana con capacidad transformadora, incorpora la comunidad científica como sujeto de esta actividad. Una disciplina consta de una estructura conceptual, un conjunto de modelos que ponen a prueba y dotan de significado al marco teórico, y una comunidad científica de profesionales que utiliza esos modelos, actúa sobre la realidad y la transforma (Kuhn, 1975).

¿Qué se entiende por educador matemático? En sentido abierto consideramos la comunidad de educadores matemáticos socialmente formada por aquel amplio grupo de personas que, de algún modo, trabajan y están en conexión con la educación matemática, a los que llamamos educadores matemáticos.

Entendemos por educador matemático a toda persona que pretende formar o instruir a otra, u otras, mediante las matemáticas, es decir, considera las matemáticas, en todo o en parte, como objeto de educación para las personas a cuya formación y desarrollo está contribuyendo (Rico \& Sierra, 1991, pp. 22). 
Los educadores matemáticos se cualifican por su actividad formadora mediante las matemáticas, con o sin referencia explícita a la investigación pero con legitimidad para contribuir a la Didáctica de la Matemática. La comunidad de educadores matemáticos tiene distintos niveles de organización y estructuración, mantiene medios propios de comunicación y difusión, organiza encuentros generales y seminarios específicos y se ocupa de profundizar, extender y difundir la cultura matemática en todos los niveles de la sociedad (Blanco, 2011; Rico, 1996). La profesión de profesor de matemáticas encuentra sus raíces y se desarrolla en la comunidad de educadores matemáticos.

\subsection{El oficio de la investigación en Didáctica de la Matemática}

Puig (1996) propone algunas precisiones para delimitar el oficio de investigador en Didáctica de la Matemática. Caracteriza la investigación en primer lugar como "indagación disciplinada con fines epistémicos". En segundo término destaca que "el oficio de investigar en didáctica de las matemáticas, en España, lo practican los profesores", subrayando que no existen grupos de expertos dedicados con exclusividad a la investigación en este campo. Este aspecto es importante ya que, cuando hablamos de investigadores en nuestra disciplina, no se puede descartar su actividad como profesores. Por ello, L. Puig precisa que la disyuntiva entre investigación y docencia no se corresponde con la disyuntiva entre teoría y práctica de la investigación: "La investigación no es la teoría de la docencia, que sería la "práctica". Hay una teoría y una práctica del oficio de investigar, como hay una teoría y una práctica del oficio de enseñar." (Puig, 1996; pp. 107-108). En los niveles docentes universitarios estas precisiones distinguen las tareas docentes e investigadoras de estos profesionales.

La consideración como investigadores de los profesores de primaria, de secundaria y de otros niveles educativos no universitarios tiene un planteamiento distinto.

En primer término, la investigación en Didáctica de la Matemática ha estado, tradicionalmente, centrada en programas de innovación curricular. La investigación y el desarrollo del currículo han de estar basados en estudios realizados en el aula y sostenerse sobre el trabajo y las experiencias de los profesores en ejercicio.

En segundo lugar, la participación de los docentes en la investigación curricular es sustantiva: "la posición clave que desempeñan los profesores en estos estudios justifica la discusión sobre su papel como investigadores. Su actividad específica está en el ámbito del diseño, la innovación o la evaluación" (Rico \& Sierra, 2000; p. 84).

En tercer lugar, sin perjuicio de su relevancia, el papel del docente en la investigación suele ser de colaboración:

"El interés del profesor suele quedar limitado por el propio interés de la innovación; no trasciende al marco teórico más amplio del cual esa experiencia forma parte. Éste es un trabajo del investigador en Didáctica de la Matemática quien, apoyándose en la colaboración del profesor, puede progresar en su esfuerzo de comprensión.” (Op. Cit. p. 86).

Profesor de matemáticas e investigador en Didáctica de la Matemática son dos profesiones fuertemente interrelacionadas, con fuertes intereses comunes, pero distintas. 


\section{Estructura de la disciplina}

El investigador novel requiere desde el comienzo de su trabajo precisar y organizar sus ideas sobre el carácter científico de la disciplina a la que se incorpora. Para atender esta necesidad revisamos algunos principios epistemológicos relativos al estatus de la investigación en Didáctica de la Matemática. Estos principios atienden la estructura de la disciplina. Los argumentos no agotan las cuestiones ni cierran sus interrogantes.

\subsection{Contextos de descubrimiento y justificación en la ciencia}

Observación y teorización son las fuentes de las que deriva el conocimiento científico. Tradicionalmente, desde el estudio de las teorías científicas, se ha manifestado que:

Todo conocimiento científico se sustenta en una doble dependencia entre los datos experimentales derivados de la observación y las formas lógicas estructuradas y necesarias. Sin los primeros no sería posible conocer nada del mundo, sin las segundas este conocimiento no podría aspirar a ser verdadero (Casares, 2009, p.1).

En este marco de filosofía de la ciencia, se enuncian dos contextos para fundamentar la ciencia: el contexto de descubrimiento y el de justificación (Reichenbach, 1938).

El contexto del descubrimiento es el tejido de circunstancias históricas y biográficas, sociales y personales, en que se produce un descubrimiento científico (...); el contexto de justificación consiste en el tejido de las relaciones lógicas entre el aserto que enuncia lo descubierto y el sistema de los conocimientos científicos ya aceptados, en particular, aquellos que suministran una demostración o confirmación de dicho aserto (Mosterín \& Torretti, 2002, p. 130).

Nuevas propuestas en filosofía de la ciencia plantearon superar la dualidad entre observación y teoría, entre descubrimiento y justificación; apostaron por una ampliación de los contextos desde los que considerar las ciencias como construcciones humanas; postularon contextos diversos para establecer los márgenes de actividad en el mundo y las posibilidades de comprensión de la realidad a través de la experimentación (Cuadrado, 2004). Los expertos denunciaron el reduccionismo establecido por H. Reichenbach. Los dos contextos mencionados resultaron insuficientes ya que ambos identificaron la actividad científica con sus resultados, la ciencia con el conocimiento científico.

\subsection{Enseñanza de la ciencia}

J. Echevarría reivindica la ciencia como actividad del hombre, que no puede reducirse a sus aspectos cognoscitivos. Interpreta la ciencia como actividad humana sostenida por cuatro contextos: "el contexto de educación enseñanza y difusión de la ciencia, el contexto de innovación, el contexto de evaluación o de valoración y el contexto de aplicación" (Echevarría, 1995, pp. 58). Este autor postula la enseñanza de la ciencia como actividad científica. Destaca el carácter necesario de la enseñanza para una formación adecuada de los científicos; subraya la regulación social de la enseñanza; remarca la importancia de la formación científica básica en la educación de los ciudadanos, singularmente presta atención a la adquisición de representaciones mentales adecuadas, sometidas a un proceso de normalización; finalmente, resalta la 
lentitud de los cambios en la enseñanza de las ciencias, para reivindicar el carácter científico de las actividades de difusión.

Esas reflexiones tienen interés especial para la Didáctica de la Matemática: "No hay descubrimiento ni justificación científica sin previo aprendizaje, por ello hay que partir del contexto de enseñanza a la hora de analizar las grandes componentes de la actividad científica [...] no hay intelección científica sin aprendizaje previo" (Echevarría, 1995, p. 59).

El contexto de enseñanza describe una actividad científica:

Incluye dos acciones recíprocas básicas, la enseñanza y el aprendizaje de sistemas conceptuales y lingüísticos, por una parte, pero también de representaciones e imágenes científicas, notaciones, técnicas operatorias, problemas y manejos de instrumentos. El individuo habrá de mostrar que tiene una competencia en el manejo de todos esos sistemas sígnicos y operatorios (p. 60).

Esta premisa es clave en nuestra aproximación: la enseñanza y aprendizaje de la matemática conforman una actividad científica y forman parte de la propia disciplina matemática. Estos argumentos establecen el estatus epistemológico de la Didáctica de la Matemática y su carácter científico.

\subsection{Investigar y resolver problemas}

Concebimos la actividad científica como una tarea de resolución de problemas. "La Ciencia es, en esencia, una actividad para [detectar, enunciar y] resolver problemas" (Laudan, 1986; p. 39). La ciencia propone dar respuesta a problemas y cuestiones reales así como transformar el mundo, no sólo el mundo físico sino también el mundo social. Investigar es un modo de construir la ciencia, de ahí que la investigación pueda considerarse como actividad de resolución de problemas. Al considerar la ciencia como acción transformadora, la investigación en Didáctica de la Matemática se presenta como tarea de resolución de problemas (Rico, 1999).

Desde una perspectiva pragmática podemos decir que una ciencia se caracteriza por aquellos problemas que plantea y resuelve. "Las materias o campos de estudio están determinadas por las clases de preguntas para cuyas respuestas han sido inventadas (...) Cada una de las grandes clases de preguntas -las fácticas y las formales- posee sus propias técnicas especializadas" (Berlin, 1983, pp. 27-28).

Un trabajo de investigación se puede analizar, en términos generales, como la resolución de un problema. Investigar implica mejorar nuestro conocimiento sobre los problemas que nos afectan y preocupan. La tarea de resolución de problemas que llamamos investigación consiste en una indagación sistemática mediante el método científico.

"El trabajo del científico comienza por la selección de un problema prometedor, un problema que sea sensible dentro de la actual situación problemática (...) la ciencia siempre empieza con problemas y termina con problemas” (Popper, 1997, p. 154).

\subsection{Resolver problemas en Didáctica de la Matemática}

Centrándonos en la Didáctica de la Matemática, sostenemos que esta disciplina se ocupa de estudiar los fenómenos de la educación matemática, investigar los problemas que se plantean y proponer marcos para su interpretación y resolución. La Didáctica 
de la Matemática se ocupa, en primer término, de un conjunto de problemas que proceden de un campo de fenómenos que surgen de la actividad humana, cuyo estudio da lugar a una disciplina organizada. La Didáctica de la Matemática trata de conjuntar herramientas de muy diversas procedencias para atender a una tarea social relevante y abordar el núcleo de su competencia: los fenómenos de transmisión, comunicación y construcción del conocimiento matemático, de los que se derivan los problemas de la enseñanza y aprendizaje de las matemáticas (Rico, 1995a).

La Didáctica de la Matemática es la disciplina dedicada al estudio racional y crítico de los problemas de la educación que surgen cuando las matemáticas son instrumento principal de formación, parte crucial del patrimonio cultural que hay que transmitir a los ciudadanos, cuando se requiere de profesionales para esta formación.

El desarrollo reciente de la Didáctica de la Matemática como disciplina científica avala su consideración como actividad de resolución de problemas. En los últimos 50 años ha sido constante destacar aquellos problemas que la disciplina aborda y resuelve en el trabajo de comités, encuentros de especialistas y debates de expertos en Didáctica de la Matemática. Freudenthal (1981) y Wheeler (1984) sistematizaron estas cuestiones y utilizaron como contraste la idea de problema en matemáticas (Rico y Sierra, 2000).

\subsection{Racionalidad crítica y principio de falsación}

Nuestro planteamiento es racionalista y pragmático. "En su forma intelectualmente más desarrollada [racionalidad] es la disposición a discutir críticamente las creencias propias y a corregirlas a la luz de las discusiones críticas con otras personas." (Popper, 1997, p. 179).

Cada ciencia es un campo que opera con términos y relaciones internas que surgen de la organización operativa de una parte de la realidad absorbida y categorizada (...) es una estrategia que se compromete operativamente con determinadas partes del mundo aislándolas y dándoles una necesidad interna que no tienen antes de ser incorporadas a un campo científico concreto (Casares, 2009, p. 8).

La actividad propia de la Didáctica de la Matemática delimita y encara la complejidad de la educación matemática, la somete a crítica para comprender y explicar sus fenómenos y para resolver sus problemas. Para ello sigue el método científico, racionaliza el conocimiento generado y evalúa y contrasta sus predicciones hasta alcanzar un conocimiento fundado. El método científico es un modo ordenado de interrogar y buscar respuestas, basado en la observación y análisis sistemático de hechos y relaciones, en la formulación de hipótesis y teorías que interpreten, expliquen, predigan o permitan actuar sobre tales hechos, y en la falsación o validación de esas hipótesis y teorías (Mosterín \& Torretti, 2002).

Entendemos aquí estas acciones y sus relaciones dialécticas en el sentido en que las utiliza Popper (1979) para el conocimiento científico. Las teorías son conjeturas sobre la naturaleza de la realidad. Forma parte del proceso consciente de construcción de teorías el que para ello utilicemos con toda libertad cualquier material: mitos, costumbres, prejuicios o suposiciones; lo importante es lo que hacemos con esas teorías, no su procedencia. Una vez formulada una teoría, debe ser severamente criticada tanto por su análisis lógico como por su contrastación empírica. 


\subsection{Ciencias humanas y ciencias de la educación}

Nos ubicamos en el ámbito de la actividad humana, en el mundo cuyos fenómenos consisten en la construcción y transmisión de conceptos y procedimientos, nos situamos entre aquellas ciencias que estudian la educación.

Schoenfeld (2000) considera que los matemáticos utilizan los términos "teoría" y "modelo" de forma diferente al uso que se hace en las ciencias de la vida y en las ciencias sociales. Para mostrarlo compara tres teorías: teoría de Ecuaciones, teoría de la Evolución y teoría de la Mente, que proceden de tres disciplinas diferentes: matemáticas, biología y psicología, y esto lo ejemplifica con tres modelos: flujo del calor en un lámina, relaciones depredador-presa y resolución de problemas, respectivamente. En todos estos casos las teorías se formulan explícitamente, los resultados se obtienen por aplicación de un método determinado, los modelos muestran aproximaciones de los fenómenos más o menos precisas a la teoría, tienen carácter explicativo y su uso consiste en hacer predicciones. Los resultados "son verificaciones empíricas de la corrección de la teoría (si bien) las teorías y los modelos en la ciencia están siempre sujetos a revisión y refinamiento" (Op. Cit. p. 5-6). La jerarquía considerada: disciplina, teoría, modelo, fenómeno, expresa el aparato teórico con el que una ciencia se organiza a partir de los problemas que aborda.

Schoenfeld sitúa las ciencias de la educación, en particular la educación matemática, dentro de un tercer grupo de disciplinas, diferenciado de las ciencias físico-matemáticas y de las ciencias de la vida. Este tercer grupo lo constituyen las disciplinas científicas formadas y formalizadas, relativas a la naturaleza humana, las ciencias humanas, donde destacan la sociología, la psicología y la antropología.

Las ciencias humanas se encuentran en proceso de constitución, en busca de objetividad.

"Piedra de toque de una ciencia que aspira a la objetividad es la presencia de dos elementos consustanciales a la investigación desde Galileo: el instrumental y la medida (...) En tanto que carezcan de instrumental que potencie y, en su caso, cuestione las frágiles y sesgadas evidencias del sentido común, algunas de las ciencias humanas o de las disciplinas dentro de ellas, no pueden alardear del carácter de científicas." (Fierro, 2011, p. 353).

La Didáctica de la Matemática es una ciencia humana en proceso de constitución.

\subsection{La investigación en Didáctica de la Matemática}

Bishop (1992) distingue tres tradiciones en investigación que configuran la disciplina Didáctica de la Matemática: la tradición normativo-interpretativa, la empírico-científica y la tradición crítica.

La tradición normativo-interpretativa, también denominada pedagógica, se ocupa de lo que sucede en el aula, trata de resolver los problemas que allí se presentan; el profesor es agente esencial en esta tradición. Destacan su metodología descriptiva, basada en el ensayo y error; trata de entender los fenómenos y refinar los datos obtenidos.

La tradición empírico-científica propone convertir la Didáctica de la Matemática en ciencia experimental, guiada por una metodología positivista que acentúe su carácter predictivo. 
La tradición crítica revisa la praxis y teoriza sobre la Didáctica de la Matemática. Su orientación es interpretativa y trata de explicar los fenómenos críticamente.

Científicos y filósofos de la ciencia han establecido criterios de demarcación para el saber científico. Las reglas metodológicas del neopositivismo constituyen el criterio más fuerte; el racionalismo crítico de K. Popper fija el método hipotético-deductivo y la falsación de teorías como ideal de la ciencia factual.

En Bishop, Clements, Keitel, Kilpatrick, y Leung (2003), Grouws (1992), Gutiérrez y Boero (2006) se presentan balances comprensivos del estado y prioridades de la investigación en Didáctica de la Matemática. Estos trabajos muestran la complejidad considerable del campo.

\subsection{Etapas en el proceso de investigación}

Los problemas que surgen en los distintos ámbitos de la enseñanza y aprendizaje de las matemáticas son objetos de estudio y trabajo para la Didáctica de la Matemática. La finalidad investigadora transforma estos ámbitos de actuación y configura la Didáctica de la Matemática como disciplina científica (Rico, Sierra, \& Castro 2002).

Considerar la investigación como una actividad de planteamiento, delimitación y resolución de problemas proporciona un modelo para el diseño y desarrollo de una investigación educativa. G. Polya (1979) integró operativamente en la resolución de problemas matemáticos el contexto heurístico, o de búsqueda y descubrimiento, con el algoritmo de resolución y justificación. Desde una perspectiva funcional consideramos momentos clave en una investigación aquellos que los expertos establecieron en el proceso de resolución de un problema. Proponemos un modelo para la realización de una investigación, cuyas etapas se basan en dichos momentos:

1. Detección e identificación de un problema.

2. Representación del problema dentro de un marco conceptual.

3. Tratamiento técnico del problema mediante diversas estrategias.

4. Explicitación de los resultados u obtención de la solución.

5. Evaluación e interpretación de los resultados (Rico, 1999).

\section{Aspectos semánticos}

"Las palabras diversamente ordenadas, constituyen diversos sentidos, y los sentidos diversamente ordenados producen diferentes efectos.” (Pascal, 1962, p. 23).

\subsection{Uso de los términos educación matemática y Didáctica de la Matemática}

Hemos precisado distintos significados atribuidos al término educación matemática y también los relativos a Didáctica de la Matemática, destacando que atienden a sentidos y modos de uso diferentes. En la literatura especializada aparecen estos términos con una diversidad y amplitud semántica considerables. J. Kilpatrick revisa la diversidad de término y usos en distintos países, que atribuye a razones culturales, académicas y prácticas. 
¿Didáctica, pedagogía o educación? En algunos países, como Francia y Alemania el término educación no es usual entre los expertos en educación matemática, quienes prefieren hablar de didáctica o de didáctica de un contenido. (...) En los países nórdicos, hay una larga tradición de designar la educación en la universidad como pedagogía de modo que los educadores matemáticos han debido disputar para que la didáctica de un contenido se reconozca como un campo de estudio genuino, parte de la pedagogía. En inglés tanto didáctica como pedagogía suelen tener una connotación peyorativa e incluso negativa, y no son usuales. (...) Parece que la terminología se escoge más por razones políticas que académicas; también parece que los patrones de uso hacen que el término educación matemática sea mas comprensivo e impreciso que el término Didáctica de la Matemática (Kilpatrick, 2008, pp. 32-33).

En Latinoamérica es usual hablar de matemática educativa como variante hispana, en lugar de educación matemática.

Los significados por los que hemos optado, si bien no coinciden con los señalados por J. Kilpatrick, enriquecen nuestro espacio de trabajo y contribuyen a la reflexión ya que se ocupan de diferenciar dos niveles: un campo de problemas que surge de procesos de transmisión cultural (educación matemática) y una disciplina que aborda el estudio de dicho campo (Didáctica de la Matemática). Los usos documentados y sus prioridades de interpretación son coherentes con los significados que hemos establecido.

\subsection{Significados para educación matemática}

En esta aproximación distinguimos tres significados para educación matemática, que corresponden a sus ámbitos de actuación.

Un primer significado es curricular. Su sentido viene dado porque se ocupa de los contextos de transmisión y evaluación del conocimiento matemático por los sistemas educativos, es decir, considera los problemas derivados de la enseñanza, aprendizaje y valoración de las matemáticas en el medio escolar.

Un segundo significado es profesional, cuyo sentido lo establecen los contextos de formación, preparación, actuación y desarrollo de los profesionales que asumen intencionalmente los procesos de enseñanza y aprendizaje (Kilpatrick, 2009).

Un tercer significado es investigador, cuyo contextos son aquellos que consideran la fundamentación y teorización para describir, interpretar, explicar, predecir y actuar sobre los fenómenos derivados de la enseñanza y aprendizaje de las matemáticas.

La investigación en Didáctica de la Matemática encuentra su objeto de estudio y trabajo en los problemas que surgen de estos tres significados. Esta investigación selecciona sus problemas en los ámbitos mencionados y se atiene a las condiciones propias de la investigación científica: indagación sistemática en un marco epistémico y con intención transformadora del medio educativo (Rico \& Sierra, 2000, p. 99).

\subsection{Significado de un concepto matemático}

Otra noción importante en nuestra aproximación es la de significado para un concepto matemático. Frege (1998) estableció la diferencia entre signo y significado de un término y, dentro del significado, distinguió entre sentido y referencia de un 
mismo término. Frege estableció que la referencia de un enunciado es su veracidad o falsedad, y su sentido es el pensamiento que expresa.

La búsqueda de la verdad es la que incita a avanzar del sentido a la referencia. El valor veritativo de un enunciado es su referencia. Cada enunciado asertivo, en el que tengan importancia las referencias de las palabras, debe ser considerado, pues, como un nombre propio y, su referencia, caso de que exista, es o bien lo verdadero o bien lo falso (Op. Cit. 34, p. 93).

En esta noción de significado de un concepto, el triángulo semántico viene dado por el signo o término con el que se expresa, por su referencia o concepto propiamente tal, y por su sentido o modo en que vienen dados los objetos que caen bajo el concepto.

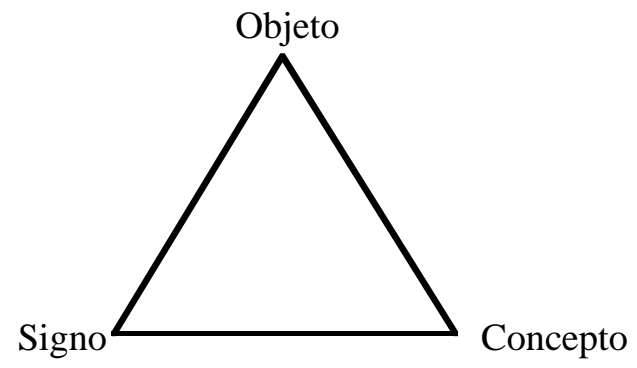

Figura 1. Triángulo semántico (concepto)

Este triángulo semántico identifica los elementos constitutivos del significado de un concepto desde una perspectiva lógica y formal. Nuestro interés por el significado está centrado en el ámbito de la matemática escolar; por ello adaptamos estas ideas a nuestro propósito y abordamos el significado de un concepto matemático en dicho ámbito atendiendo a tres dimensiones: los sistemas de representación, la estructura conceptual y la fenomenología (Rico, citado por Gómez, 2007, pp. 23-27).

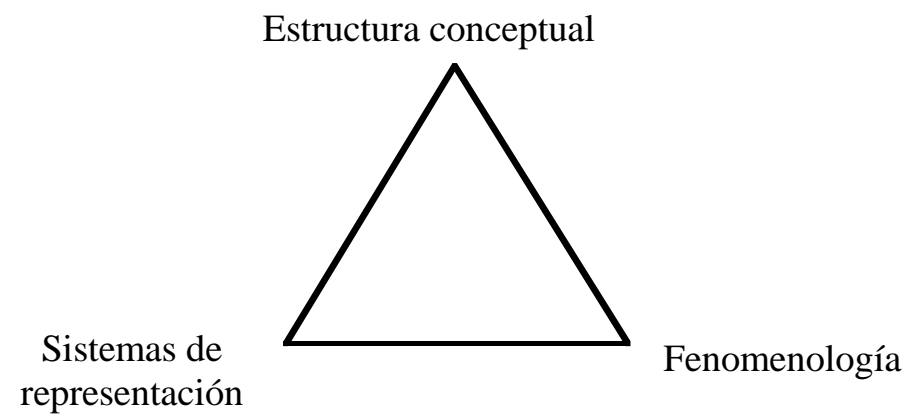

Figura 2. Triángulo semántico de un concepto matemático escolar

Tres componentes determinan el significado de un concepto matemático en educación:

- Los sistemas de representación, definidos por los conjuntos de signos, gráficos $\mathrm{y}$ reglas que hacen presente dicho concepto y lo relacionan con otros.

- La estructura conceptual, que comprende conceptos y propiedades, los argumentos y proposiciones que se derivan y sus criterios de veracidad. 
- La fenomenología, que incluye aquellos fenómenos (contextos, situaciones o problemas) que están en el origen del concepto y le dan sentido.

\section{El conocimiento}

Todo conocimiento científico se sustenta en una doble dependencia entre los datos experimentales derivados de la observación y las formas lógicas estructuradas y necesarias. Sin los primeros no sería posible conocer nada del mundo, sin las segundas este conocimiento no podría aspirar a ser verdadero (Casares, 2009, p. $1)$.

\subsection{Conocimiento matemático}

Hemos argumentado que la enseñanza y aprendizaje de una disciplina constituyen parte de su actividad como ciencia. Desde esta premisa, sostenemos que la Didáctica de la Matemática hace parte del contexto educativo de la matemática, donde encuentra su fundamento.

Conceptos y estructuras de las matemáticas escolares provienen de las disciplinas matemáticas. Clave, por tanto, para la Didáctica de la Matemática es el modo en que se consideran las matemáticas. Matemáticos y educadores matemáticos lo hacen de manera diferente. Para el matemático, las matemáticas se basan en la prueba y la demostración; se establece como ciencia de la cantidad y del espacio, de la estructura y las relaciones; es la ciencia que llega a conclusiones necesarias (Kilpatrick, 2008).

Las matemáticas consisten en enunciados que formulan proposiciones, hipótesis o afirmaciones, que pueden ser verdaderos y cuya verdad se puede obtener a partir de proposiciones primitivas. La educación matemática se ocupa de los términos en cuanto expresan conceptos significativos, cuyo significado se puede inferir a partir de definiciones desde conceptos primitivos.

Los problemas que se plantean al educador matemático son problemas de comprensión y de uso, problemas de significado, no consisten en pruebas y demostraciones. El educador matemático centra su interés en los significados de los conceptos y estructuras de las matemáticas escolares. Para ello, el profesor de matemáticas comienza por considerar distintas representaciones de un mismo concepto; analiza sus propiedades y las relaciones que establece con aquellos otros que forman parte de la misma estructura, sus enunciados y proposiciones; examina los fenómenos que están en su origen, de los cuales procede. También contempla la dimensión histórica de los conceptos matemáticos que analiza. La historia de las matemáticas, de los cambios que un determinado concepto ha experimentado, aporta información valiosa para su enseñanza y aprendizaje.

Los educadores tienen un planteamiento comprensivo por razón de que las cuestiones implicadas afectan a cómo las matemáticas se aprenden, a cómo se aplican y cómo se usan. Para ello, hace emerger los significados de los conceptos centrales de las matemáticas escolares. A su vez, el investigador en Didáctica de la Matemática observará los fenómenos de comprensión de los escolares, el modo en que los datos experimentales muestran los aprendizajes, es decir los modos de significar del aprendiz en el proceso de construcción de nuevos conocimientos. 


\subsection{Lo educativo y la Didáctica de la Matemática}

La dimensión educativa lleva a considerar el conocimiento matemático como una actividad social, propia de los intereses y la afectividad del niño y del joven, cuyo valor principal está en que organiza y da sentido a una serie de prácticas útiles, a cuyo dominio hay que dedicar esfuerzo individual y colectivo. La sociología del conocimiento establece que, como en el resto de las disciplinas científicas, las representaciones matemáticas son construcciones sociales. El conocimiento matemático, como todas las formas de conocimiento, representa las experiencias materiales de personas que interactúan en entornos particulares, culturas y periodos históricos. El educador se ocupa de iniciar a los niños y adolescentes en la cultura de la comunidad a la que pertenecen y de transmitirles sus valores sociales; de esta cultura también forma parte el conocimiento matemático, que debe transmitirse en toda su plenitud a cada generación. La responsabilidad del educador matemático es grande puesto que [las matemáticas] son una herramienta intelectual potente, cuyo dominio proporciona privilegios y ventajas intelectuales. La tarea del educador matemático debe ofrecer respuestas para la multiplicidad de opciones e intereses que surgen y se entrecruzan permanentemente en el mundo actual (Rico, 1995b).

\subsection{Investigación en Didáctica de la Matemática}

Schoenfeld (2000) destaca dos fines principales para la investigación en educación matemática: uno como ciencia básica, que consiste en comprender la naturaleza del pensar, enseñar y aprender matemáticas. Un segundo, como ciencia aplicada, que consiste en emplear tal comprensión para mejorar la instrucción en matemáticas. Ambas finalidades las considera fuertemente interrelacionadas, tan importante una como otra.

Como disciplina, la Didáctica de la Matemática tiene su campo de estudio en ese complejo de actividades de significación y tareas sociales de formación que hemos denominado educación matemática. La Didáctica de la Matemática se ocupa de indagar metódica y sistemáticamente sobre la enseñanza y aprendizaje de las Matemáticas así como proporcionar fundamentación teórica y sostener los planes para la cualificación profesional de los educadores matemáticos.

Hemos delimitado tres campos de reflexión en la educación matemática, que consideran problemas distintos y proceden de ámbitos de actuación diferenciados.

Un primer campo, el curricular, se centra en la transmisión del conocimiento y en su evaluación, es decir, estudia los problemas derivados de la enseñanza, aprendizaje y valoración de las matemáticas en el medio escolar.

Un segundo campo, el profesional, contempla la formación, preparación, actuación y desarrollo de quienes asumen la responsabilidad de los procesos de enseñanza y aprendizaje.

El tercer campo, el científico, considera la actividad de fundamentación, teorización y experimentación que permite interpretar, predecir y actuar sobre los fenómenos derivados de la enseñanza y aprendizaje de las matemáticas.

La investigación en Didáctica de la Matemática estudia y trabaja sobre los problemas que se detectan y surgen en estos tres campos. Selecciona sus problemas en los ámbitos mencionados y se atiene a las condiciones propias de la investigación 
científica: indagación sistemática en un marco epistémico y con intención transformadora del medio educativo (Rico \& Sierra, 2000).

Los fenómenos sobre los que trabajan los investigadores en Didáctica de la Matemática contemplan las relaciones de comunicación, los modos de entender y conocer, los procesos de construcción y los actos de comprensión que se expresan mediante la cantidad, la regularidad, la forma y otras estructuras matemáticas. Por esta razón el marco teórico para la Didáctica de la Matemática se establece sobre cuatro dimensiones o campos disciplinares. Sostenemos que el marco teórico de la Didáctica de la Matemática se articula sobre cuatro dimensiones: pedagógica, cognitiva, conceptual y sociológica (Rico, 1997). El marco teórico en que se plantean, estudian e investigan los problemas y cuestiones de la Didáctica de la Matemática se sostiene desde el dominio de la Matemática, la Pedagogía, la Psicología y la Sociología de la Educación. No cabe confusión entre las cuestiones prioritarias de estas disciplinas y las de la Didáctica de la Matemática: se distingue que un problema es propio de este área de conocimiento cuando están implicados procesos de comunicación, enseñanza y aprendizaje de las matemáticas. Postulamos que se sostiene en las disciplinas citadas de manera específica, que trasciende e integra las aportaciones concretas de cada una de ellas (Rico \& Sierra, 2000).

\section{El método}

"El método científico consiste en la observación y el análisis sistemático de hechos, formulación de hipótesis y teorías y comprobación de su validez. De ahí que investigar consista en la indagación sistemática, controlada, empírica y crítica de proposiciones hipotéticas acerca de las relaciones presumidas entre fenómenos naturales, basada en el método científico." (RAC, 1990).

Un trabajo de investigación en Didáctica de la Matemática se delimita según tres componentes: su encuadre teórico, su marco metodológico y su ámbito de actuación.

\subsection{Indagación enfocada en problemas}

El desarrollo del conocimiento comienza por corregir un conocimiento anterior. Respecto a la Didáctica de la Matemática, conviene interpretar sus teorías como intentos de encontrar respuesta a distintos problemas que se plantean en la disciplina. Popper (1997) sintetiza el método científico en cuatro pasos:

1. Seleccionar un problema.

2. Tratar de resolverlo proponiendo una hipótesis derivada de una teoría, como solución tentativa.

3. Discusión crítica de la teoría, según el poder predictivo de la hipótesis que de tal teoría emana, para eliminar algunos errores; comprensión de nuestros problemas y teorías; necesidad de nuevas soluciones.

4. Sacar a la luz nuevos problemas mediante la discusión crítica.

De estas categorías, K. Popper afirma que la eliminación de errores a través de la crítica es la más característica. La crítica de una teoría científica es siempre un intento de encontrar y eliminar un error, una falla en la teoría.

"Una de las formas de crítica mas elocuente e importante es el recurso a la observación, el experimento y la medición. Si podemos mostrar que las consecuencias de una teoría no 
son compatibles con ciertos hechos, tenemos un argumento poderoso contra ella (...) las observaciones, los experimentos y las mediciones sólo son interesantes en el contexto de la discusión crítica de alguna teoría." (Popper, 1997, pp. 157-160).

En toda investigación subyace una revisión y crítica a las soluciones parciales aportadas en estudios previos.

\subsection{Fases en una investigación}

El investigador novel comienza por la detección e identificación de un problema, que sitúa en uno de los ámbitos de la educación matemática. Trata de precisar el problema que quiere estudiar, la cuestión que le preocupa, los fenómenos que quiere observar, los sujetos con los que va a trabajar, las operaciones o procedimientos que va a seguir para poner a prueba una hipótesis y la institución donde va a llevar a cabo el estudio. Esta elección general responde a alguna cuestión surgida en su trabajo profesional dentro de la educación matemática. Progresivamente, el investigador expresa con mayor claridad las intuiciones iniciales y señala los puntos débiles que tienen hasta el momento sus interpretaciones. De este modo perfila el problema que quiere estudiar y surge la necesidad de ubicarlo dentro de un marco.

En segundo lugar, la representación del problema debe estar basada en un marco teórico. Este momento transcurre con la caracterización del problema que se quiere estudiar, con la consecución de un enunciado que exprese una conjetura discutible, cuyo significado venga dado por un marco conceptual. Se pueden seguir distintas técnicas, como revisar bibliografía, buscar en bases de datos, seleccionar documentos que hayan abordado previamente el problema. Finalmente, conviene elaborar un informe sobre el estado de la cuestión y resultados de investigación relacionados.

Forma parte del proceso de representación del problema el enunciado de sus objetivos generales y, junto con él, de las hipótesis o conjeturas que se quieren abordar.

El científico observa con instrumentos y método, pone a prueba las conjeturas, en ocasiones experimenta. La ciencia se sustenta en la observación metódica de la realidad. El método científico consiste en observar y razonar para explicar y predecir. Por ello, en tercer lugar, el investigador novel realiza el trabajo de campo para el estudio escogido. Diseña un modelo con el cual poner a prueba la conjetura provisional enunciada. En este momento el investigador actúa según el diseño metodológico, que aporta una guía y proporciona unas técnicas específicas.

Pasos importantes de esta etapa son la delimitación del medio y de las fuentes de información sobre las que va a trabajar; la definición y elaboración de instrumentos para la recogida de datos; variables que se consideran; categorías para su estudio; la organización, codificación y técnicas para el tratamiento de los datos; asimismo tienen interés los ensayos piloto, que permiten la revisión de tareas e instrumentos.

A partir de las observaciones llegamos a la obtención de resultados. Los resultados de una investigación son las explicaciones de las observaciones dentro de un marco teórico determinado. Actividad importante es la interpretación general y específica de los datos obtenidos, según criterios y categorías previamente establecidos y la toma de decisiones si procede. La conexión vuelve a centrarse en el marco teórico, ya que datos y observaciones deberán encontrar explicación dentro de un marco para que se puedan considerar como resultados, y mostrar su potencia explicativa en relación con el problema inicialmente planteado. 
Por último hay una evaluación e interpretación final de los resultados, donde la información obtenida se debe ajustar a un marco explicativo global con el que abrir nuevas perspectivas para el avance en la investigación. Este es un momento clave ya que permite una interpretación amplia del problema y una respuesta a las conjeturas formuladas. En este momento se conectan las tres componentes de la investigación: ámbito de actuación, marco teórico y marco metodológico. El estudio manifiesta su auténtico alcance cuando destaca su ubicación en un plan general e incluye una reflexión global y abierta hacia nuevos interrogantes.

Junto con las conclusiones hay que destacar los problemas que quedan abiertos, cuestiones surgidas durante el estudio que no se contemplaban en el planteamiento del problema, vinculación del estudio con otros campos no considerados inicialmente (Rico, 1999).

\subsection{Paradigmas e instrumentos}

Indagar con método científico implica tener una orientación paradigmática y metodológica determinada. Kilpatrick y Sierpinska (1993), consideran tres aproximaciones metodológicas generales de la investigación educativa: positivista, fenomenológica y crítica, aproximaciones que revisan las tradiciones consideradas por Bishop (1992). Después de analizar estos tres paradigmas estos autores adoptan una posición holística respecto a los métodos de investigación educativa: "no existe un único método de investigación que pueda abarcar todo el rango de preguntas que interesan a los educadores matemáticos." (Op. cit. p. 17). La orientación metodológica en Didáctica de la Matemática la enmarcan los paradigmas positivista, interpretativo y crítico. Estas tradiciones se muestran en Cohen, Manion, y Morrison (2011).

Desde el paradigma neo-positivista todo cuanto existe, existe en cierta cantidad y, por tanto, puede ser medido. Experimentar constituye un modo preferente de medición y estudio para el investigador en Didáctica de la Matemática, siempre y cuando sea viable y sirva de manera eficaz y eficiente al objetivo de la investigación. En este paradigma las técnicas e instrumentos, cualquiera que sea su desarrollo, artesanal, técnico o tecnológico, deben satisfacer requerimientos obligatorios que permitan ajustar la observación, calibrarla, hacerla objetiva y desligarla de las impresiones del investigador individual.

La información y el conocimiento cualitativos proporcionan un primer nivel de conocimiento, descriptivo e insuficiente a largo plazo como conocimiento científico. La cuantificación constituye objetivo imprescindible para desarrollar una ciencia predictiva.

Con un planteamiento cercano a la metodología de investigación Sierra (2011), a su vez, destaca cinco tipos de investigación en Didáctica de la Matemática. Proporciona una caracterización de cada uno de esos planteamientos, que sintetiza como: investigación descriptiva o de examen, investigación experimental, investigación cualitativa o interpretativa, investigación histórica, e investigación acción. 


\subsection{El método de análisis}

Por análisis se entiende, con carácter general, un método o conjunto de métodos que resuelven lo complejo en lo simple. La segunda regla del Discurso del Método propone: "dividir cada una de las dificultades que examinare en cuantas partes fuere posible y en cuantas requiriese su mejor solución" (Descartes, 2011, p. 114).

En Didáctica de la Matemática distinguimos por su uso en la investigación tres niveles de análisis: conceptual, de contenido y didáctico (Rico \& Fernández-Cano, 2012).

\section{Análisis conceptual}

Una de las mayores dificultades de la investigación en ciencias sociales y, por tanto, de la investigación en las disciplinas educativas es la enorme polisemia de buena parte de los conceptos centrales que se utilizan en esos estudios. (...) Como conocimiento científico los conceptos son públicos, son el tipo de cosas que muchas personas pueden compartir y comparten. La atribución arbitraria de significado o la desconsideración de significados centrales, son usos patológicos de los conceptos que han de quedar excluidos del trabajo científico. (...) El análisis conceptual se preocupa por la naturaleza de las definiciones y del lenguaje; trata de encuadrar los términos y sus interconexiones. Examina cuidadosamente la diversidad de significados, las posibilidades de conexión entre los términos y los niveles subjetivos (creencias y concepciones) y objetivos (conceptos) de cada campo conceptual. Contextualiza la definición dentro del área en que se inserta. Usa ejemplos y contraejemplos, en vez de la definición explícita. Emplea analogías y términos evocativos en vez de pruebas, axiomas o cuantificaciones (Rico, 2001, pp. 185-186).

\section{Análisis de contenido}

Cohen, Manion y Morrison (2011) señalan que

"el término análisis de contenido indica el proceso de recogida y resumen de datos escritos los contenidos principales de dichos datos y sus mensajes. De modo más preciso, [el análisis de contenido] define un conjunto de procedimientos estricto y sistemático para el análisis riguroso, el examen y verificación de los contenidos de datos escritos. (...) El análisis de contenido se puede llevar a cabo con cualquier tipo de material escrito, desde documentos a transcripciones de entrevistas, desde productos de los media hasta entrevistas personales. Se utiliza frecuentemente para analizar un número considerable de textos, debido a su naturaleza sistemática, gobernado por reglas; también porque permite utilizar el análisis asistido por ordenador. Utiliza la categorización como rasgo esencial para reducir grandes cantidades de datos" (Op. Cit., p. 563).

La Tabla 1 muestra la comparación entre análisis conceptual y análisis de contenido, según lo utilizamos para la investigación en Didáctica de la Matemática. 
Tabla 1: Comparación entre el análisis conceptual y el análisis de contenido

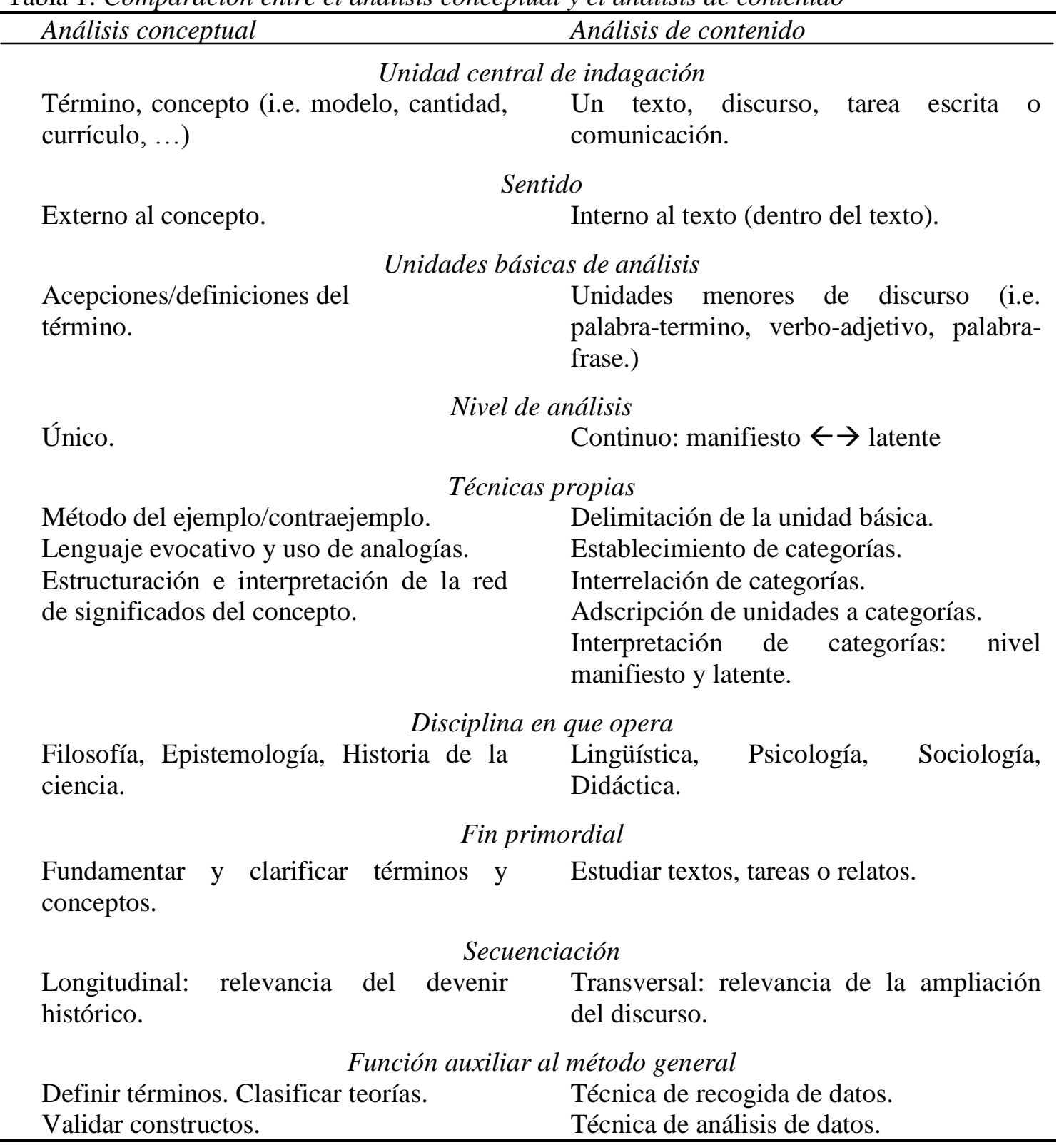

\section{Análisis didáctico}

La noción de análisis didáctico aporta un método de trabajo y reflexión para el estudio ordenado de los problemas didácticos, singularmente los problemas de la Didáctica de la Matemática. Calificamos el análisis como didáctico cuando sus procedimientos antes descritos se centran en entender e interpretar la estructura, la organización, el comportamiento y las conexiones de los procesos de enseñanza y aprendizaje o los procesos de formación de profesores, tal y como se emplean en los conocimientos, normas, argumentos, textos y relatos educativos. Por ello los procesos generales considerados se refieren, explícita o implícitamente, a la enseñanza y aprendizaje de las matemáticas escolares o a la formación de sus profesores.

El análisis didáctico se considera integrado por análisis de distintos tipos. Estos tipos de análisis se diferencian por las categorías que emplean, categorías que hemos denominado organizadores y que se ajustan a las dimensiones conceptual, cognitiva, formativa y social con la que hemos estructurado la noción de currículo (Rico, 1997). 
En Rico, Marín, Lupiáñez, y Gómez (2008) se detalla el análisis didáctico del sistema de los números naturales como un ejemplo.

\section{Conclusión}

Las ideas que aquí se han expuesto son producto de la reflexión y de la práctica llevadas a cabo con grupos de graduados en las dos últimas décadas que se han iniciado a la investigación en Didáctica de la Matemática. Los planteamientos, discutidos suponen un avance para la investigación en educación matemática, ya que han aportado propuestas, detectado carencias y subsanado errores. En cada caso la bibliografía remite a un tratamiento más detallado de las cuestiones presentadas.

Los argumentos propuestos e ideas organizadas atienden al lenguaje, la estructura, el conocimiento, el significado y el método en Didáctica de la Matemática. Proceden de la experiencia adquirida en la formación de investigadores en la disciplina. Se han difundido en seminarios y encuentros nacionales e internacionales (Rico, 1996; Rico, 2000; Rico, Fernández-Cano, Castro, \& Torralbo, 2008), dos congresos del ICMI (Rico, 1998; Rico, 2008), y otros informes y estudios comparativos (Hart y Hitt, 1999; Rico, 2004; Vallejo, Fernández-Cano, Torralbo, \& Rico, 2007).

Singularmente, se han concretado en el curso Investigación en Educación Matemática. Avances Metodológicos, materia impartida por el autor de este trabajo en el Programa de Tercer Ciclo para el Doctorado en Didáctica de la Matemática de la Universidad de Granada desde el bienio 1994-1996 hasta el bienio 2006-2008, en colaboración con los doctores Castro y Fernández-Cano. Con posterioridad a las adaptaciones de los estudios de posgrado del año 2007 esta materia, ubicada en el Programa del Máster Didáctica de la Matemática de la Universidad de Granada, se ha desarrollado hasta el momento en los cursos 2008-2009, 2009-2010, 2010-2011 y 2011-2012 (Rico, Perales, \& Gómez, 2011).

\section{Referencias}

Berlin, I. (1983). Conceptos y categorías. Un ensayo filosófico. México: Fondo de Cultura Económica.

Bishop, A. (1992). International perspectives on research in mathematics education. En D. Grouws (Ed.), Handbook of research on mathematics teachig and learning. New York: Macmillan.

Bishop, A., Clements, K., Keitel, C., Kilpatrick, J. \& Leung, F. (Eds.) (2003). Second International handbook of mathematics education. Roterdam: Kluwer.

Blanco, L. (2011). La Investigación en educación matemática. Educatio Siglo XXI, 29 (1), $109-128$.

Casares, A. (2009). Lógicas y contextos en la construcción de las ciencias. A Parte Rei. Revista de Filosofía, 65, 1-19.

Cohen, L., Manion, L., \& Morrison, K. (2011). Research methods in education. Londres: Routledge.

Cuadrado, G. (2004). Los contextos de la ciencia. Influencias y consecuencias. Proyecto Leonardo. Revista de Ciencia y Tecnología 1(0), 6-10.

Descartes, R. (2011). Discurso del método. M. García Morente (Trad). Madrid: Gredos.

Echevarría, J. (1995). Filosofía de la ciencia. Madrid: Akal. 
Fierro, A. (2011). Humana ciencia. Del ensayo a la investigación en la Edad Moderna. Barcelona: Anthropos.

Frege, G. (1998). Sobre sentido y referencia. En L. M. Valdés (Ed.), Ensayos de semántica y filosofía de la lógica (pp. 84-111). Madrid: Tecnos.

Freudenthal, H. (1981). Major problems of mathematics education. Educational Studies in Mathematics, 12(2), 133-150.

Gómez, P. (2007). Desarrollo del conocimiento didáctico en un plan de formación inicial de profesores de matemáticas de secundaria. Granada: Universidad de Granada.

González, W. (2010). La predicción científica. Madrid: Montesinos.

Grouws, D. (1992). Handbook of research on mathematics teaching and learning. New York: MacMillan.

Gutiérrez, A., \& Boero, P. (2006). Handbook of research on the psychology of mathematics education. Past, present and future. Rotterdam: Sense Publishers.

Hart, K., \& Hitt, F. (Eds). (1999). Supervisión of higher degrees in mathematics education. An international perspective. México: CINVESTAV.

Kilpatrick, J. (2008). The development of mathematics education as an academic field. En M. Menghini, F. Furinghetti, L. Giacardi, \& F. Arzarello (Eds.), The first century of the International Commission of Mathematical Instruction (1908-2008). Reflecting and shaping the world of mathematics education (pp. 25-39). Roma: Istituto della Enciclopedia Italiana fondata da Giovanni Treccani.

Kilpatrick, J. (2009). The mathematics teacher and curriculum change. PNA, 3(3), 107-121.

Kilpatrick, J., \& Sierpinska, A. (1993). What is research in mathematics education and what are its results? Recherches en Didactique des Mathématiques, 13(1.2), 191-204.

Kuhn, T. (1975). La estructura de las revoluciones científicas. México DF: Fondo de Cultura Económica.

Laudan, L. (1986). El progreso y sus problemas. Madrid: Encuentro Ediciones.

Mead, M. (1985). Educación y cultura en Nueva Guinea. Barcelona: Paidós.

Mosterín, J., \& Torretti, R. (2002). Diccionario de lógica y filosofía de la ciencia. Madrid: Alianza Editorial.

Niss, M. (1998). Aspects of the nature and state of research in Mathematics Education. Roskilde: IMFUFA.

Pascal, B. (1962). Pensamientos. Madrid: Espasa- Calpe.

Polya, G. (1979). Cómo plantear y resolver problemas. México DF: Trillas.

Popper, K. (1979). El desarrollo del conocimiento científico. Conjeturas y refutaciones. Buenos Aires: Paidós.

Popper, K. (1997). Modelos, instrumentos y verdad. En K. Popper, El mito del marco común. En defensa de la ciencia y la racionalidad (pp. 153-179). Barcelona: Paidós.

Puig, L. (1996). La didáctica de las matemáticas como tarea investigadora. En L. Puig, \& J. Calderón (Eds.), Investigación y didáctica de las matemáticas, (pp. 63-75). Madrid: Ministerio de Educación y Ciencia.

Real Academia de Ciencias Exactas, Físicas y Naturales (1990). Vocabulario científico y técnico. Madrid: Espasa.

Real Academia Española (1992). Diccionario de la lengua española. Madrid: Espasa- Calpe.

Reichenbach, H. (1938). Experience and prediction. Chicago: University of Chicago Press. 
Rico, L. (1995a). Didáctica de la Matemática como campo de problemas. En E. Repetto, \& G. Marrero (Eds.), Estrategias de intervención en el aula desde la LOGSE (pp. 551-579). Las Palmas: ICEPSS Editores.

Rico, L, (1995b). Conocimiento numérico y formación del profesorado. Discurso de apertura, Curso Académico 1995-96. Granada: Universidad de Granada.

Rico, L. (1996). Formación de investigadores en educación matemática: el programa de doctorado de la Universidad de Granada. En L. Puig, \& J. Calderón (Eds.), Investigación y Didáctica de la Matemática (pp. 75-88). Madrid: Ministerio de Educación y Ciencia.

Rico, L. (Coord.) (1997), La educación matemática en la enseñanza secundaria. Barcelona: Horsori.

Rico, L. (1998). Programas de investigación doctorales y académicos en educación matemática en las universidades españolas. En A. Alsina, J. Alvárez, B. Hodgson, C. Laborde, A. Pérez, L. Rico, \& A. Sfard (Eds.), 8th International Congress on Mathematical Education. Selected Lectures (pp. 369-389). Sevilla: SAEM Thales.

Rico, L. (1999). Development of Spanish doctoral studies in didactic of mathematics. En K. Hart y F. Hitt (Eds.), Supervisión of higher degrees in mathematics education. An international perspective (pp. 1- 26). México: CINVESTAV.

Rico, L. (2000), Educación Matemática, investigación y calidad. En J. Ponte, \& L, Serrazina (Eds.). Educação Matemática em Portugal, Espanha e Itália (pp. 303-312). Lisboa: Secção de Educação Matemática da Sociedade Portuguesa de Ciencias de Educação.

Rico, L. (2001) Análisis conceptual e investigación en Didáctica de la Matemática. En P. Gómez, \& L. Rico (Eds.), Iniciación a la investigación en Didáctica de la Matemática. Homenaje al profesor Mauricio Castro (pp. 179-193). Granada: Universidad de Granada.

Rico, L. (2008). Formation of researchers in mathematics education, DG9. En M. Niss (Ed.), Proceedings of the 10th International Congress on Mathematical Education (pp. 461464). Denmark: Roskilde University.

Rico, L., \& Sierra, M. (1991). La comunidad de educadores matemáticos. En A. Gutiérrez (Ed.), Área de conocimiento Didáctica de la Matemática (pp. 11-58). Madrid: Síntesis.

Rico, L., \& Sierra, M (2000). Didáctica de la Matemática e Investigación. En J. Carrillo \& L. C. Contreras (Eds.), Matemática española en los albores del siglo XX, (pp. 77-131). Huelva: Hergué Editores.

Rico, L., Sierra, M. \& Castro, E. (2000). La Didáctica de la Matemática. En L. Rico \& D. Madrid (Eds.), Fundamentos didácticos de las áreas curriculares (pp. 351-406). Madrid: Editorial Síntesis.

Rico, L., Sierra, M. y Castro, E. (2002). El área de conocimiento Didáctica de la Matemática. Revista de Educación, 328 35-58.

Rico, L., \& Fernández-Cano, A. (2012). Análisis didáctico y metodología de investigación. En L. Rico, J. L. Lupiáñez, \& M. Molina (Eds.), Análisis didáctico en Educación Matemática. Granada: Universidad de Granada, pendiente de publicación.

Rico, L., Fernández-Cano, A., Castro, E. \& Torralbo, M. (2008). Postgraduate study program in Mathematics Education at the University of Granada (Spain). En E. Reys, \& J. Dossey (Eds.), U. S. Doctorates in mathematics education: developing stewards of the discipline. (pp. 203-211). Providence, R.I.: American Mathematical Society, CBMS Issues in Mathematics Education.

Rico, L., Marín, A., Lupiáñez, J. L., \& Gómez, P. (2008). Planificación de las Matemáticas Escolares en Secundaria. El caso de los números naturales. Revista SUMA, 58, 7-23. 
Rico, L., Perales, F. J., \& Gómez, P. (2011). Guía docente asignatura Investigación en Educación Matemática, avances metodológicos. Departamento de Didáctica de la Matemática. Master en Didáctica de la Matemática, Curso 2011- 2012, Universidad de Granada. Disponible en http://masteres.ugr.es/didacticamatematica/

Schoenfeld, A. (2000). Propósitos y métodos de investigación en Educación Matemática. Notices of the American Mathematical Society, 47(3), 641-649.

Seco, M., Andrés, O. \& Ramos G. (1999). Diccionario del español actual. Madrid: Aguilar.

Sierpinska, A. \& Kilpatrick, J. (1998). Mathematics Education as research domain: A search for identity. An ICMI Study. Roterdamm: Kluwer Academic Publishers.

Sierra, M. (2011) Investigación en educación matemática: objetivos, cambios, criterios, métodos y difusión. Educatio Siglo XXI 29(2), 173- 198.

Vallejo, M., Fernández-Cano, A., Torralbo, M. \& Rico, L. (2007). History of spanish mathematics education focusing on $\mathrm{PhD}$ Theses. Internacional Journal of Science and Mathematics Education, 6(2), 313-327. Disponible: http://www.springerlink.com/content/q560520387q15374/?p=bfcd25c8cbf646caa66c168 64e9643fa\&pi $=7$

Wheeler, D. (1984). Research in mathematics education. For the Learning of Mathematics, 4 (1), 40-47, 4(2), 39-44, y 4(3) 22-29.

\section{Referencia del autor}

Luis Rico Romero. Facultad de Ciencias de la Educación. Universidad de Granada (España).

lrico@ugr.es 